\title{
The Architecture of the Access Protocols of the Global Infocommunication Resources ${ }^{\dagger}$
}

\author{
Natalya Verzun ${ }^{1, * \mathbb{D}}$, Mikhail Kolbanev ${ }^{1,2} \mathbb{D}$ and Alexey Shamin ${ }^{2} \mathbb{D}$ \\ 1 Saint Petersburg Electrotechnical University “LETI”, Professor Popov Str. 5, 197376 St. Petersburg, Russia; \\ mokolbanev@mail.ru \\ 2 Nizhny Novgorod State University of Engineering and Economics, Oktyabrskaya Str. 22a, \\ 606340 Knyaginino, Russia; ngiei-spo@mail.ru \\ * Correspondence: verzun.n@unecon.ru \\ $+\quad$ This paper is an extended version of our report: Verzun, N.; Kolbanev, M.; Vorobeva, D. “Model of \\ a Centralized Strategy for Selecting the Last Mile Access Network" in the Majorov International \\ Conference on Software Engineering and Computer Systems (MICSECS 2019), Saint-Petersburg, Russia, \\ 12-13 December 2019.
}

Received: 1 May 2020; Accepted: 1 June 2020; Published: 9 June 2020

check for updates

\begin{abstract}
One of the important functions of cyberspace is to provide people and devices with access to global infocommunication resources, and as the network infrastructure develops, the number of access options increases, including those based on wireless technologies. A wide variety of access technologies leads to the formation of heterogeneous broadcast networks. Following the concept of Always Best Connected and striving for rational use of access network resources, developers use Vertical Handover procedures today. This approach assumes the existence of a selection criterion that allows you to prefer a particular network to other networks from the number of available and able to provide the required connection and services, and a selection procedure that implements the process of calculating the characteristics of access in each of the acceptable options. When implementing a vertical handover, it should be taken into account that the rational choice depends on the moment of time and point of space at which the terminal device developed a request to establish a connection. The corresponding procedures can be implemented in accordance with decentralized or centralized architectures. In the first case, the choice is made by hardware and software of terminal devices. The disadvantage of this implementation is the complexity and, as a result, the rise in price of terminal devices, each of which requires a corresponding complexity of the selection procedure of the performance and memory reserve. Another negative consequence of the decentralized approach is a decrease in the last-mile network utilization rate due to the inability to make complex decisions. The article discusses the centralized architecture of access protocols to global infocommunication resources. In accordance with it, the access network is selected by a new centralized network device that was not previously used on communication networks. The protocols that this network element implements should be located between the first (physical) and second (channel) levels of the open system interaction model. The purpose of the study is to develop an effective architectural solution for access networks and create a mathematical model for evaluating the efficiency of using the last mile resources and the quality of user service. The object of research is architectural solutions for last-mile networks. The subject of research is models of the theory of tele-traffic that allow us to evaluate the qualitative characteristics of the corresponding process. To achieve this goal the following tasks were solved in the article: analysis of known approaches to selecting one of several available access networks; development of a centralized architecture that changes the basic model of interaction between open systems; description of the metadata exchange scenario between network elements of the new architecture; development of a mathematical model of the data transmission process in the access radio network; conducting numerical estimates of the probabilistic and temporal characteristics of the proposed procedures.
\end{abstract}


Keywords: heterogeneous network; vertical handover; network architecture; access network; access scenario; models for evaluating the probability-time characteristics of access

\section{Introduction}

This article is based on the materials of the report that was made by the authors at MICSES-2019 in December 2019 and published in proceedings of MICSES-2019 [1].

An important purpose of cyberspace is to support the ability of people and devices to access global infocommunication resources. Since the beginning of our century, there has been an appearance and growth in the number of different options for accessing infocommunication resources, due to the development of network infrastructure.

Implementation of radio access to global infocommunication resources at the last mile according to [2] is carried out using a variety of multiple access procedures that allow rational use of a common channel resource limited by the spectrum of the radio channel.

The wide range of access radio networks available to the terminal device at the last mile [2] made it possible for the terminal to refuse at any time and at any point in space from an already established network connection, and switch to using the resources of another access radio network that is more suitable for transmitting current traffic. Furthermore, the terminal device can simultaneously communicate with multiple radio networks of access and use of their network resources.

The totality of all access networks available at a certain point in space, each of which can function using a particular radio technology, forms a heterogeneous broadcast network. In order to efficiently manage the available resources of a heterogeneous network, as well as to comply with the concept of Always Best Connected (ABC) [3], developers today use a Vertical Handover. Vertical Handover (VHO) [4] is a procedure for transmitting support for a terminal device traffic transfer session from one access radio network to another access radio network, regardless of the location of the device.

Performing the vertical handover procedure in a heterogeneous network in practice requires solving several interrelated problems [5]:

- Accumulation, consolidation, storage and analysis of all data required for making an informed decision about the vertical handover procedure;

- Selecting the appropriate access radio network from several currently available ones;

- Connecting the terminal device to the new radio network (without losing the existing communication session).

The purpose of the study presented in [1] was to develop a centralized strategy for managing access to network resources at the stage of selecting the best radio network for access to infocommunication resources. The object of research is a set of all access radio networks available at the last mile to the terminal device, forming a heterogeneous terrestrial network. The subject of the research is mathematical models for evaluating the probabilistic and temporal characteristics of the process of transmitting user data in the radio network at the last mile.

In [1], the following tasks were considered and solved, in particular:

- Analysis of existing solutions used in practice to implement procedures for selecting a suitable access radio network;

- A new, centralized strategy for selecting the access radio network is proposed and a scenario for interaction between terminal devices, aggregating devices, and access radio networks is developed;

- Developed a mathematical model of the process of information interaction on the last mile in the case of using a centralized strategy and selecting a radio access network.

The essence of the proposed strategy was that the architecture of a heterogeneous access network is supplemented by an aggregation device (AD) or otherwise an aggregator of access radio networks, 
which performs functions related to the centralized selection of the access radio network on the last mile. At the same time, access to global infocommunication resources is carried out in two steps:

I-Select an access network.

II-Transfer of user data.

A mathematical model of step II was proposed. When developing the model, the use of Time Division Multiplexing (TDM) technology for organizing multiple access to the transmission channel was envisaged. The mathematical model is a Queuing model in discrete time and includes two phases, each of which has the form $M^{d} / G^{d} / 1$.

In this article in contrast with [1]:

- First, the emphasis is on developing the architecture of protocols for interaction between terminal devices and the base station. The proposed architecture is an extension of the previously known and developed Institute of Electrical and Electronics Engineers architecture described in the 802 group of standards [6].

- Secondly, the problem of studying the impact of traffic restriction procedures at the first stage of access on the probability-time characteristics of the data delivery process from the terminal devices of the base station is solved.

The peculiarity of the developed architecture, protocols, scenario and mathematical model of information interaction of the network component in the last mile is that they are focused on providing access services to global infocommunication resources of various terminal devices: both smartphones (people) and smart things, as provided in the 5th generation networks and Network 2030 [7].

\section{Materials and Methods}

In practice, network operators often use different solutions based on a decentralized approach to select the appropriate last-mile access radio network. In this case, the selection task is solved by each terminal device separately. This study suggests another alternative approach to selecting a last mile access radio network-a centralized approach based on the implementation of a heterogeneous access network architecture of the AD-access network aggregator (see Figure 1). An AD is an auxiliary network centralized hardware and software device that is an intermediate element that connects terminal devices with the base stations of access radio networks.

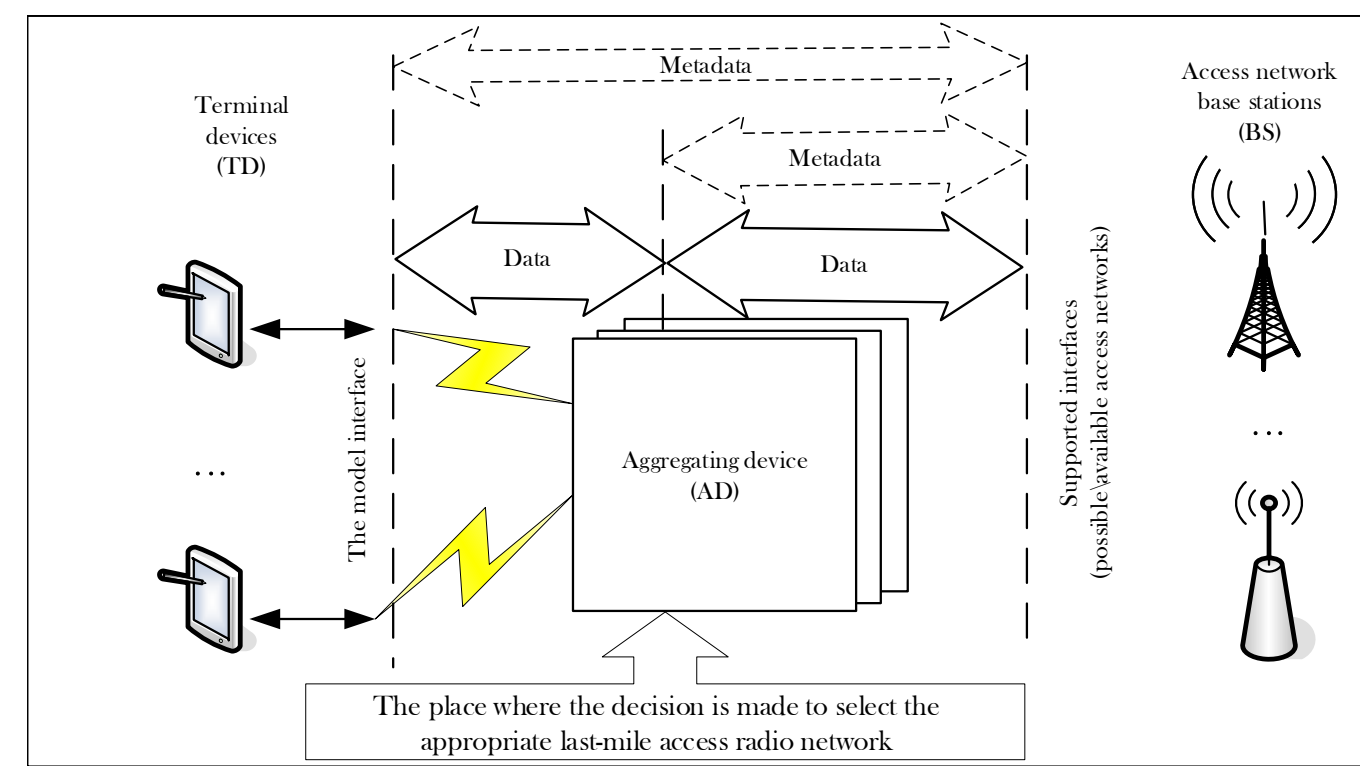

Figure 1. Centralized approach to selecting the last mile access radio network.

The access network aggregator is designed for: 
- Implementation of last-mile access radio network selection;

- Support the transfer of blocks of user data between the interfaces, terminal devices and interfaces in radio networks the last mile.

The aggregating device is shared by many terminals to access network resources. This architecture can be called a sharing access architecture. In the sharing architecture (Figure 2) the transmission media access control (MAC) layer is divided into two sublevels:

- Sublevel of the selection of access network;

- Sublevel of access to the transmission medium.

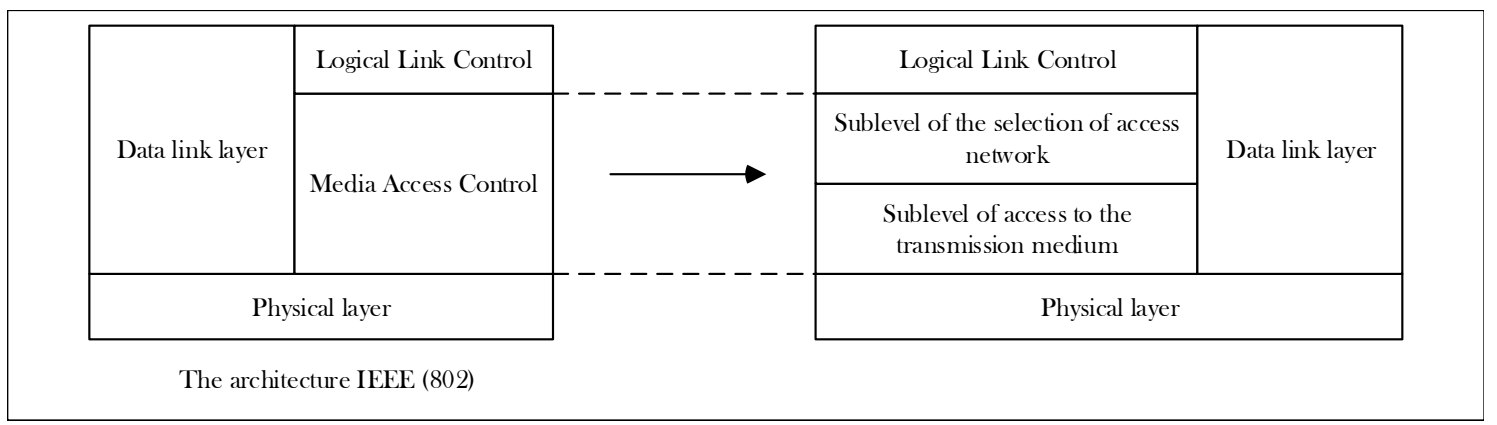

Figure 2. Architecture of device access protocols to global infocommunication resources on the last mile.

According to this architecture, the last-mile resource access service scenario is implemented in two steps:

I-Selecting the radio network to access,

II-Transmitting user data.

A graphical representation of the access procedure is shown in Figure 3.

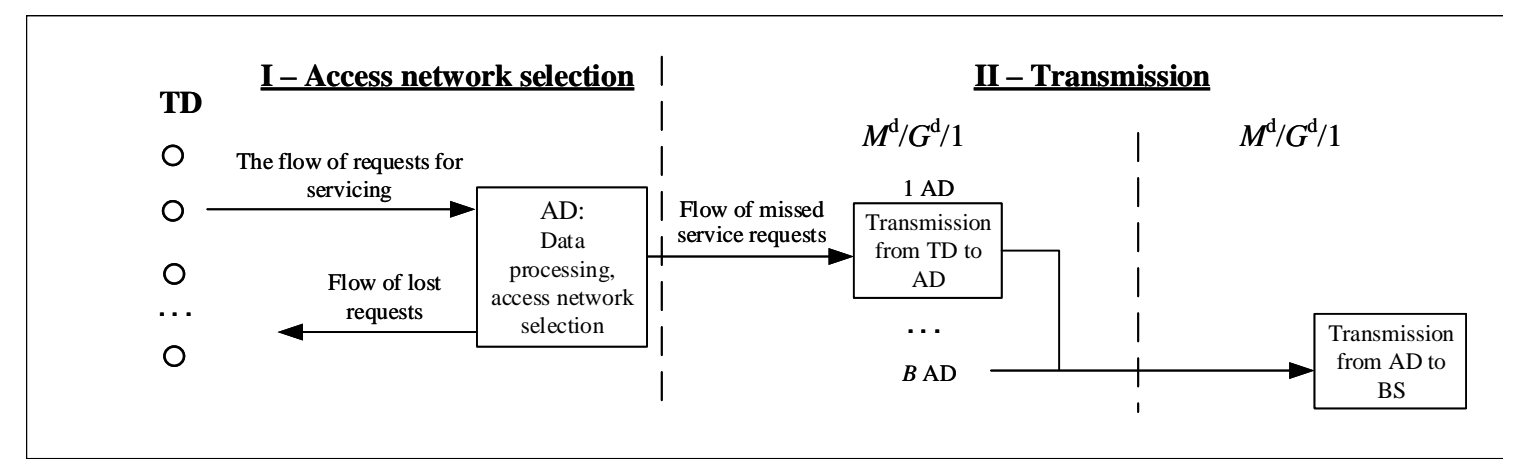

Figure 3. Visual description of step-by-step organization of access to global infocommunication resources.

\subsection{Step I. Selecting the Radio Network to Access}

At the first step, a request for a specific infocommunication service is received from the terminal device to a centralized aggregating device. The aggregating device, based on data about the quality of service requested by the terminal, weighs the possibility of providing the service, and also selects the most appropriate radio network for this request.

If the resources available at the moment to provide the requested service are not enough, or they are not sufficient to maintain the required level of transmission quality, the terminal device is refused to provide the requested service.

If a suitable radio network is selected and its resources are sufficient, the required network resources are reserved for the entire transmission section from the terminal device to the base station.

After that, we move to the second step in providing access to information resources data transfer. 
The procedure for selecting the most appropriate access radio network for providing the service is not considered in this study. It is assumed that this procedure can be carried out based on the analysis of collected and accumulated historical data characteristics established in a heterogeneous broadcast network of connections, as well as characteristics related to the terminal device and transmission quality requirements due to the type of service requested. For example, different groups of metrics can be analyzed that characterize:

- Quality of data transmission in the access radio network;

- Terminal devices;

- $\quad$ Resource requirements that depend on the type of telecommunications service;

- Subscriber profile;

- $\quad$ Requirements for safety and reliability.

Evaluation of the effectiveness of information and communication systems can be carried out using traditional indicators, which are the essence of the concept of Quality of Service, QoS. These are, for example: network performance characteristics: bandwidth of the communication channel, probabilistic and temporary characteristics of the information transfer process, information transfer speed [8], reliability and security characteristics: fault tolerance, availability coefficient $[9,10]$.

The list of traditional and long-used indicators used for monitoring the effectiveness of information and communication systems can be expanded with alternative indicators. For example, in [11,12], probabilistic and energy indicators that depend on the technical characteristics of terminal devices and on the spatial parameters of access radio networks are considered.

\subsection{Step II. Data Transfer from the Terminal Device to the Base Station}

The process of transferring data blocks from the terminal device to the base station is represented by a two-phase queuing system (see Figure 3):

1 Simulates the process of transferring user data blocks from/to terminal devices to/from the radio network aggregator;

2 Simulates the process of transmitting user data blocks from/to the aggregator of access radio networks to/from the base station of the selected radio network.

The following notation will be used later in the article:

$\mu \quad$ Intensity of the flow of user data units coming from a single terminal device [1/s];

$D \quad$ The number of terminal devices that are within the range of a single radio network aggregator;

$B \quad$ Number of radio network aggregators;

$N \quad$ Number of access radio networks;

$l \quad$ Length [bits] of user data blocks to transmit;

$P \quad$ Probability of receiving a refusal to provide the service at the Ist step;

$Q=1-P \quad$ Probability of getting permission to provide the service at step I;

$V_{1}, V_{2} \quad$ Bit rate on phase 1 and 2 [bit/s];

$T_{a 1}, T_{a 2}$ Mathematical expectation of the allowed time of information aging at phase 1 and 2, respectively $[\mathrm{s}]$.

Consider that:

- At the output of each terminal device for transmitting over the network access to the base station a uniform simple stream of user data blocks is formed $l$ [bit]. Flow intensity $\mu[1 / \mathrm{s}]$ and it is a Bernoulli flow with the parameter $q_{1}$ on interval $T_{1} \cdot T_{1}$ which we will use as the minimum clock cycle for simulating the transfer of user data blocks in phase $1 T_{1}=1 / V_{1}$ [s]; 
- At the output of each radio network aggregator for transmission to the base station, a stream of uniform user data blocks for transmission is formed, which form a Bernoulli-type stream with the parameter $q_{2}$ on interval $T_{2}$ which we will use as the minimum clock cycle for simulating the transfer of user data blocks in phase $2 T_{2}=1 / V_{2}$ [s];

- Direct transmission of user data blocks is performed (i.e., feedback is not provided). Transmitted user data blocks that were damaged in phase 1 and 2 when transmitting over the radio channel are discarded when receiving;

- In the 1st and 2nd phases of the second step of the scenario, multiple access of terminal and aggregating devices to the transmission medium is implemented using TDM technology.

\subsubsection{Mathematical Model}

This section offers a mathematical model of the process of transferring user data blocks at the second step of the scenario under consideration - the transfer from the terminal device to the base station. When developing the mathematical model, the features of the physical and channel level were taken into account (see Figure 2).

In general, the mathematical model is represented by an expression for the z-transformation of a series of time distribution delays in transmitting user data blocks:

$$
g(z)=g_{1}(z) g_{2}(z)
$$

where $g_{1}(z)$ and $g_{2}(z)$ are $z$-transformations of time distribution series for delay of transmission of user data blocks on the 1st and 2nd phases, respectively.

Taking into account the above assumptions both phases of transmission are presented in the form of QS $M^{d} / G^{d} / 1$ in discrete time.

Formulas that specify the form $g_{1}(z)$ and $g_{2}(z)$ at each transmission phase have the form:

$$
\begin{gathered}
g_{j}(z)=\frac{\left(1-\theta_{i}\right)(1-z) f_{j}(z)}{1-p_{j} z-q_{j} z f_{j}(z)}, p_{j}=1=q+j, j=\overline{1,2} \\
q_{1}=\mu Q T_{1} ; q_{2}=\left(\overline{\Pi_{1}} \mu Q D T_{2}\right) / N
\end{gathered}
$$

where $\overline{\Pi_{1}}$ - probability that the user data block in the first phase will be delivered in a timely manner, $\theta_{1}$-the probability that the transmitting buffer of the terminal device is busy during the first transmission phase (i.e., it has at least one block to transmit); $\theta_{2}$-the probability that the transmitting buffer of the network aggregator in the second transmission phase is busy. $\theta_{1}$ and $\theta_{2}$ are defined according to the formula:

$$
\theta_{j}=\left.q_{j}\left(d / d z^{-1}\right) f_{j}(z)\right|_{z=1}{ }^{\prime} \theta_{j}<1, j=\overline{1,2} .
$$

$f_{j}(z)$-z-transformations of service interval distribution series on the $j$-th transmission phase $(j=\overline{1,2})$, the following parameters based on the TDM application are set:

$$
f_{1}(z)=z^{-l D} ; f_{2}(z)=z^{-l B}
$$

The final expressions for $\theta_{1}$ and $\theta_{2}$, if we consider (4), may be like:

$$
\theta_{1}=q_{1} l D, \theta_{2}=q_{2} l B .
$$

The mathematical model of the process of transferring user data blocks in the second step of the scenario under consideration-the transfer from the terminal device to the base station-is represented by Equations (1)-(6). 


\subsubsection{Expressions for Calculating Probability-Time Characteristics (PTC)}

The probability that the user data blocks being transmitted will be delivered in a timely manner.

The study assumes the situation of using a stochastic restriction on the allowed time for transmitting user data blocks in the 1st and 2nd phases with the parameter $Q_{a j}$ :

$$
Q_{a j}=1-\frac{T_{j}}{T_{a j}}, j=\overline{1,2}
$$

In this situation, you can use the following formula to find the probability that the transmitted user data blocks will be delivered in a timely manner:

$$
\bar{\Pi}=\overline{\Pi_{1}} \overline{\Pi_{2}}, \overline{\Pi_{j}}=\left.g_{j}(z)\right|_{z=Q_{a j}^{-1}} j=\overline{1,2},
$$

where $g_{j}(z)$ is set by Equations (2) and (3).

The average delay time for transmitting user data blocks from the terminal device to the base station can be found from the expression:

$$
\bar{t}=\overline{t_{1}}+\overline{t_{2}}
$$

where $\overline{t_{1}}$ and $\overline{t_{2}}$ are mathematical expectations for the transfer times of user data blocks in phase 1 and 2 are based on the Khinchin-Polachek formula

$$
\begin{gathered}
\overline{t_{j}}=\overline{n_{j}} T_{j}, \overline{n_{j}}=f_{j}^{\prime}(1)+\frac{q_{1} f_{j}^{\prime \prime}(1)}{2\left(1-\theta_{j}\right)}, j=\overline{1,2}, \\
f_{j}^{\prime}(1)=\left.\left(d / d z^{-1}\right) f_{j}(z)\right|_{z=1} f_{j}^{\prime \prime}(1)=\left.\left(d / d z^{-2}\right) f_{j}(z)\right|_{z=1^{\prime}} j=\overline{1,2},
\end{gathered}
$$

where $f_{j}(z)$ is set by Equation (5).

The information speed of the real time access radio network at the last mile is determined from the expression

$$
R^{R T}=l D \mu Q \bar{\Pi},
$$

where $\bar{\Pi}$ is set by Equation (8). $R^{R T}$ shows how much [bit] traffic is successfully transmitted in the access radio network per unit of time [s]. The $R^{R T}$ calculation takes into account all losses that occur when transmitting user data blocks on the last mile section. The losses are caused, first, by possible service failures at the first step (see Figure 3), and, secondly, losses arising from delayed delivery of user data blocks in the 1st and 2nd phases of the second step-directly in the transfer process.

\subsubsection{Numerical Experiments}

The following numerical data were selected for numerical experiments: $D=100 ; 200, l=256$ [bit], $B=4, N=5, V_{1}=20[\mathrm{Mbit} / \mathrm{s}], V_{2}=80[\mathrm{Mbit} / \mathrm{s}], T_{a 1}=T_{a 2}=50[\mathrm{~ms}]$.

Figures 4-9 show graphs illustrating the results of numerical experiments. The influence of the number of terminal devices in the radio network $D=100 ; 200$ and the probability of access to service (i.e., to the IInd step) $(Q=1 ; 0.9 ; 0.7)$ on the PTC of the process of transmitting user data blocks in the radio network was studied.

Figure 4-dependence of the probability that the transmitted user data blocks will be delivered in a timely manner on the intensity of receiving blocks for transmission.

Figure 5-the dependence of the average transmission time of user data blocks on the intensity of their receipt for transmission.

Figure 6-the dependence of the information speed of the real-time access radio network at the last mile on the intensity of receipt of user data blocks for transmission. 


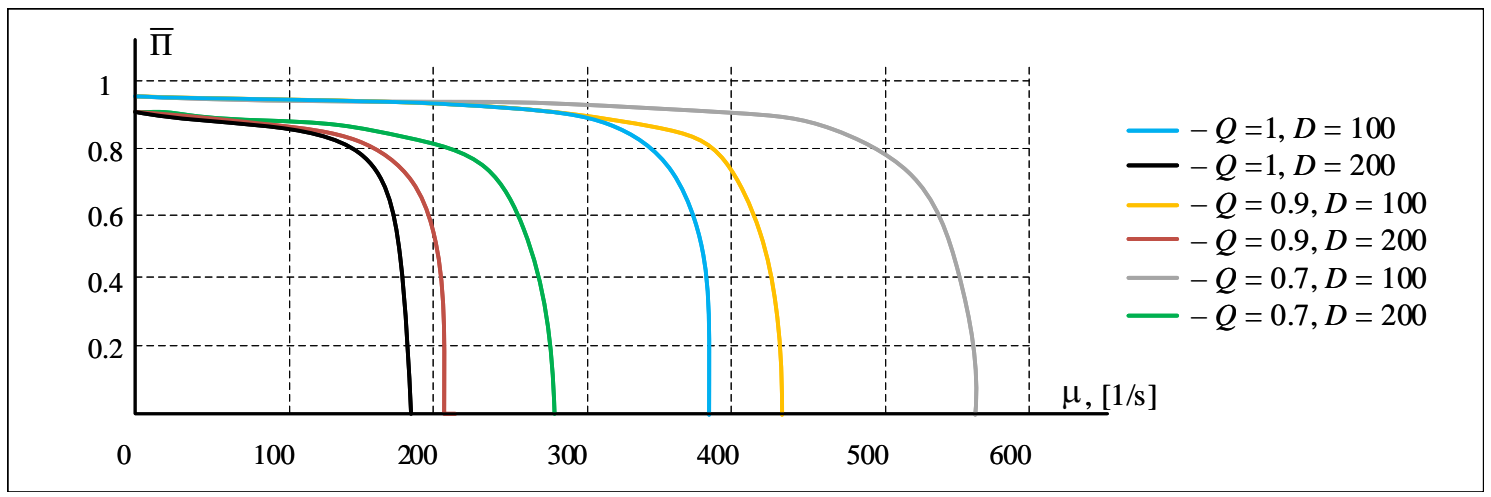

Figure 4. Dependence of the probability that the transmitted user data blocks will be delivered in a timely manner on the intensity of receiving blocks for transmission.

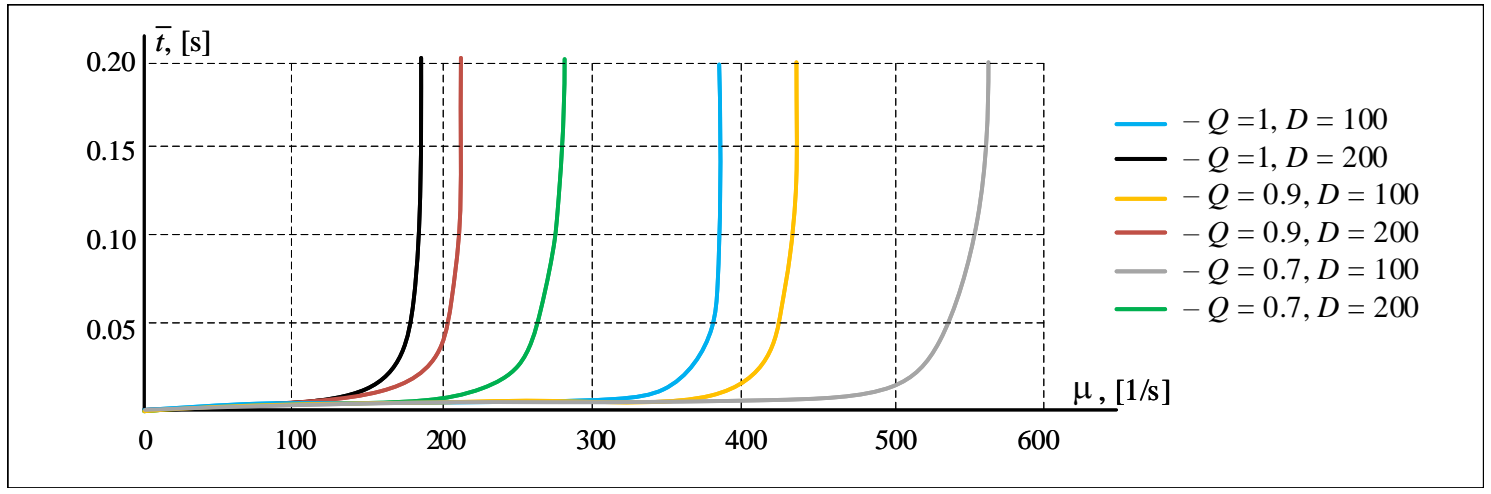

Figure 5. The dependence of the average transmission time of user data blocks on the intensity of their receipt for transmission.

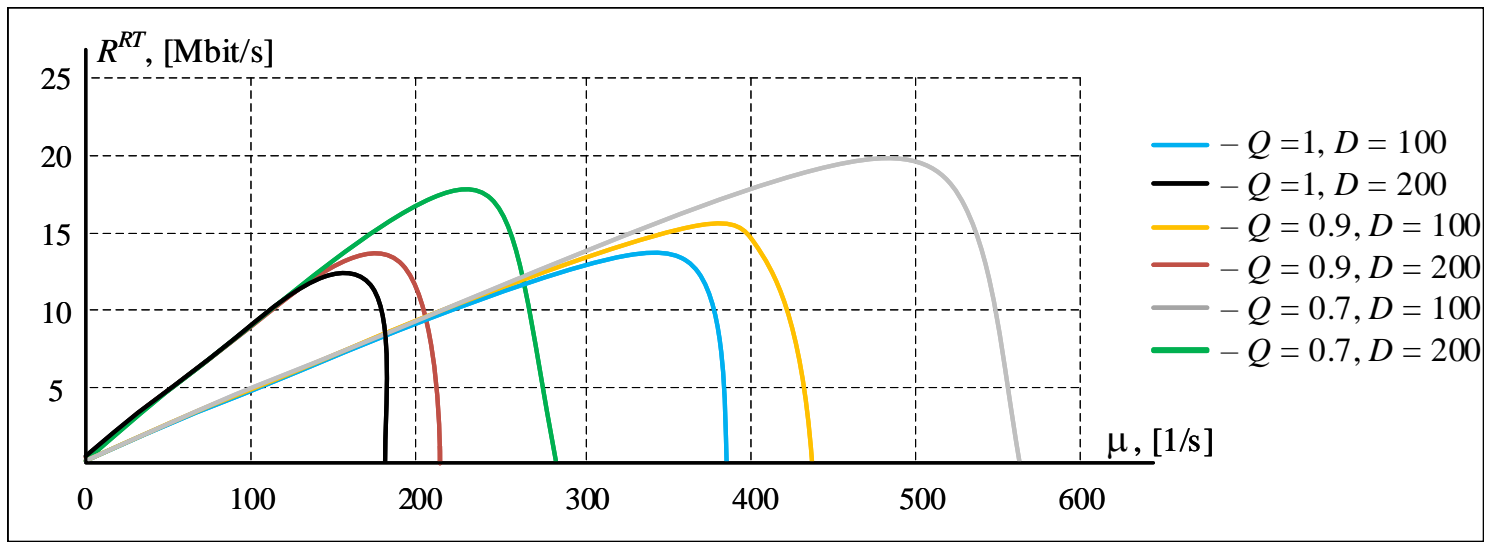

Figure 6. The dependence of the information speed of the real-time access radio network at the last mile on the intensity of receipt of user data blocks for transmission.

The dependency graphs (Figures 4-6) show that an increase in the number of terminal devices, as well as the intensity of the appearance of user data blocks for transmission (i.e., an increase in the load on the radio network) leads to a noticeable deterioration of the studied characteristics of the transmission process. So, if the procedure for dropping part of the traffic at the 1st service step is not applied (i.e., $Q=1$ )), if there are 100 working terminal devices in the access radio network, the range of operating intensities: $\mu=0 \ldots 380[1 / \mathrm{s}]$. If the number of working devices is increased by two times, this range is reduced by almost two times: $\mu=0 \ldots 180[1 / \mathrm{s}]$.

However, the introduction of a procedure for sifting (discarding) part of the traffic at the 1st stage of maintenance can significantly improve the PTC of transmission and increase the operating range of intensities. So, for the case described above, if you set the probability of access to service $Q=0.9$, 
then at 100 operating terminals, the range of operating intensities: $\mu=0 \ldots .430[1 / \mathrm{s}]$, and when their number increases by two times: $\mu=0 \ldots 220[1 / \mathrm{s}]$.

Thus, we can say that the introduction of a procedure for sifting part of the traffic allows you to increase the stability of the access radio network to increase the load.

The dependence of the probability-time characteristics of the process of transmitting user data blocks on the probability of denial of service $(P)$ at different values of the intensity of receipt of user data blocks for transmission was studied $(\mu=100 \ldots 250[1 / \mathrm{s}])$.

Figure 7-graph of the probability that the transmitted user data blocks will be delivered in a timely manner versus the probability of a denial of service.

Figure 8-graph of the dependence of the average transmission time of user data blocks on the probability of failure in service.

Figure 9-graph of the dependence of the information speed of the real-time access radio network on the last mile on the probability of failure in service.

From the dependency graphs shown in Figures 7-9, it can be seen that the introduction of a traffic screening procedure is advisable to use when the load on the network increases.

From Figure 9 it should be noted that when considering the source data, it is necessary to enter a procedure for sifting part of the traffic, only when $\mu>200[1 / \mathrm{s}]$. Furthermore, the higher the intensity of input flows of user data blocks, the greater the probability of failure $(P)$ should be selected for the stable operation of the access radio network. So, for stable operation of the radio network when $\mu=200[1 / \mathrm{s}]$ it is advisable to discard at least $10 \%$ of traffic, and when $\mu=225[1 / \mathrm{s}]$ no less than $20 \%$.

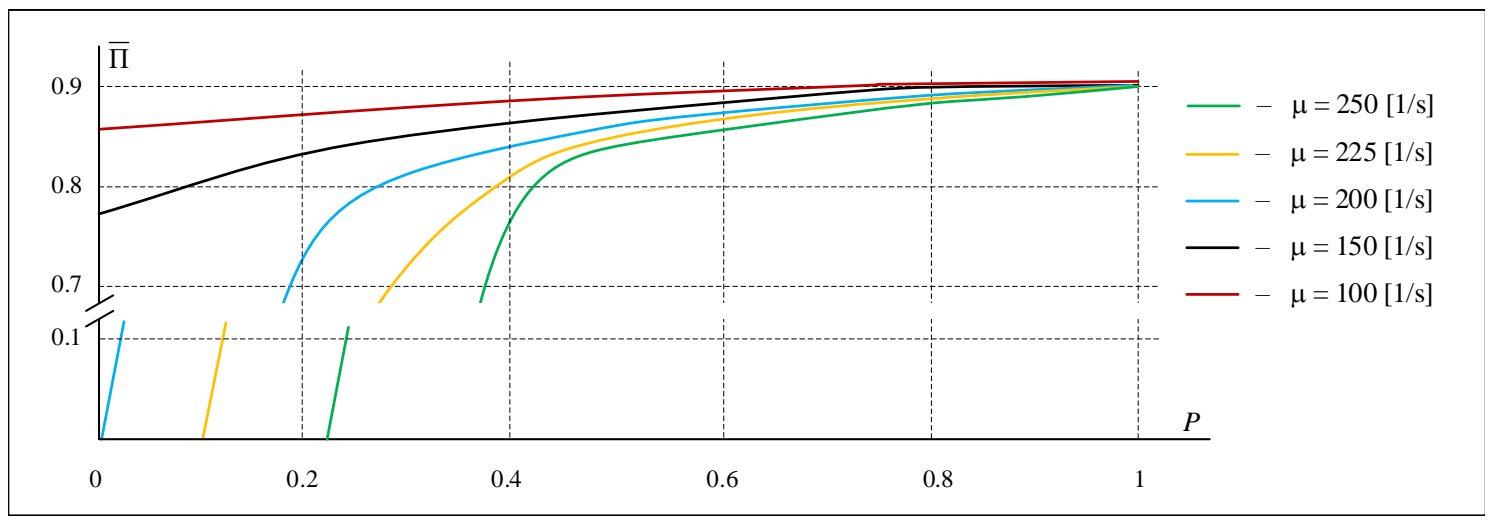

Figure 7. The probability that the user blocks being transmitted will be delivered in a timely manner depends on the probability of a denial of service.

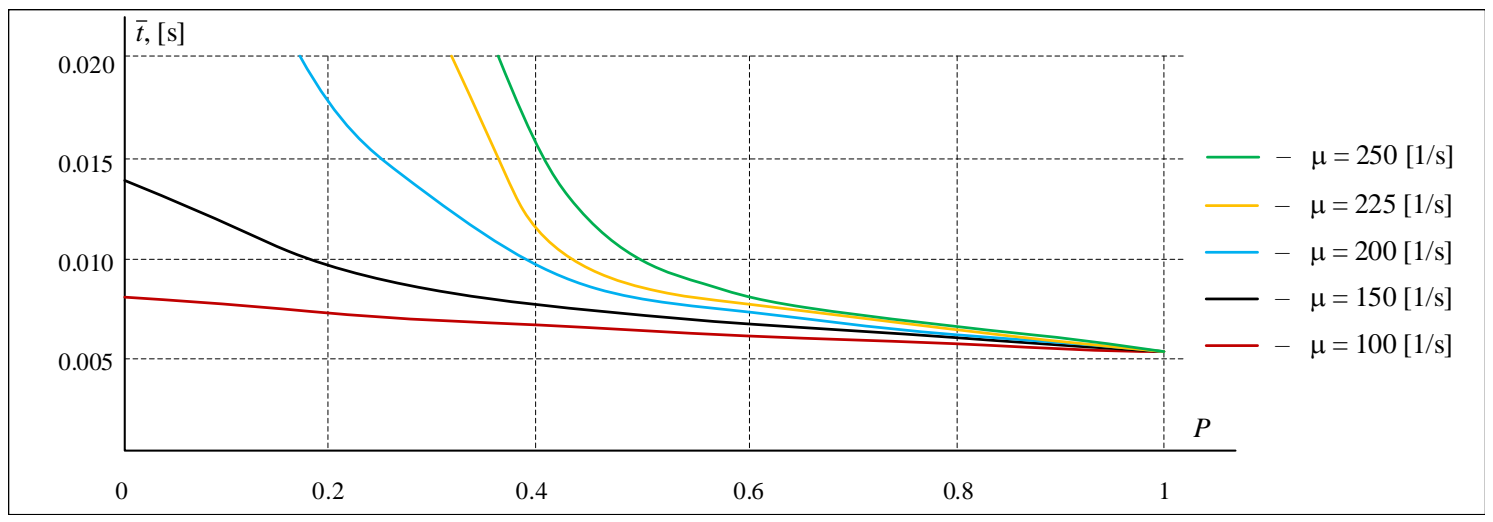

Figure 8. The dependence of the average transmission time of user data blocks on the probability of failure in service. 


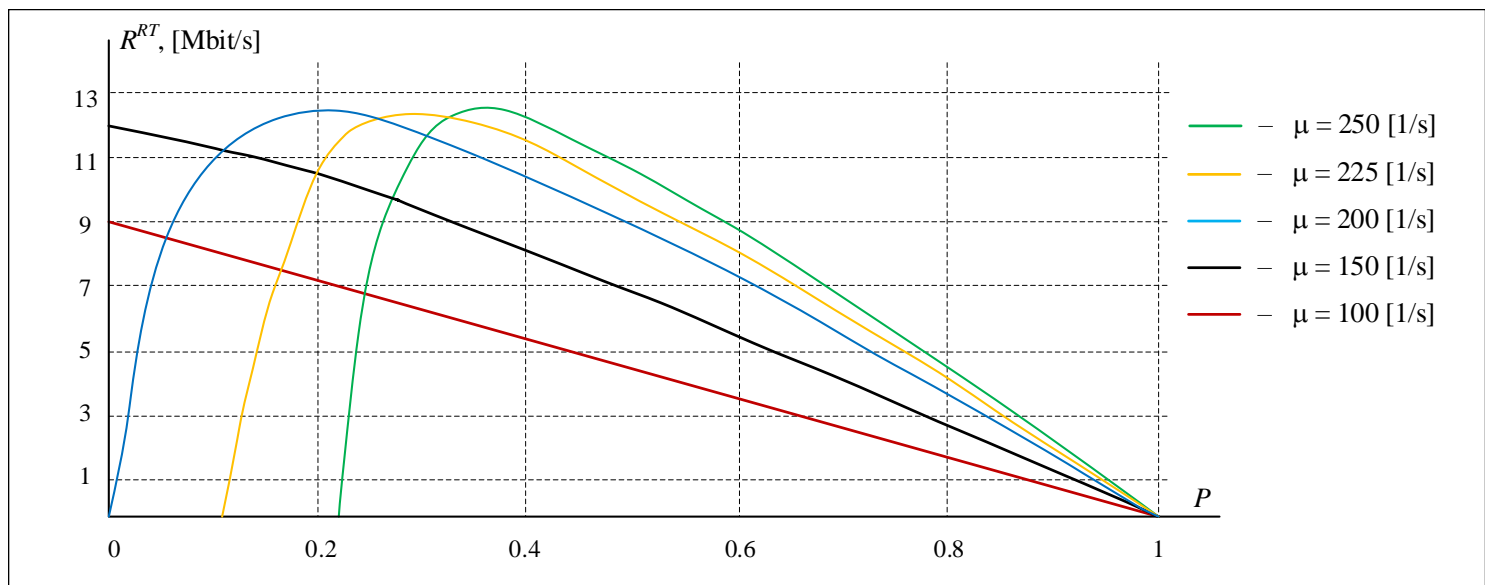

Figure 9. The dependence of the information speed of the real-time access radio network on the last mile on the probability of denial of service.

By varying the value of the access probability at the first service step, taking into account the current intensity of user data blocks appearing for transmission, you can achieve optimal network load and prevent network overload situations (loss of ergodicity).

In a traditional decentralized architecture, terminal access equipment and programs $[13,14]$ are used inefficiently, only during service receipt. In the proposed centralized sharing architecture, it is possible to programmatically manage the use of the $\mathrm{AD}$ and the quality of user service. In fact, numerical experiments show that a new mechanism for adaptive network management appears at the last mile.

\section{Results}

1. The developed centralized access network architecture differs from the known ones by using special protocols located between the physical and channel levels of the reference model of open systems interaction, which allows solving the problems of vertical handover using a new network element-a hardware and software aggregator, simplifying the schemes of terminal devices and increasing the use of networks in the last mile.

2. The sharing architecture of access protocols to global infocommunication devices has been developed, which provides for splitting the access level to the transmission medium (MAC) at the last mile into two sublevels: the sublevel of access network selection and the sublevel of access to the transmission medium. At the selection sub-level, a comprehensive selection of wireless networks from among those that meet the quality of service requirements is implemented.

3. A queuing model is obtained that describes the process of interaction between network terminals and the base station through a centralized network element in the form of a two-phase QS, which allows to evaluate the PTC of the data circulation process between the terminals and the base station.

4. Numerical experiments were carried out to assess the impact of the data block transfer process on the access network of the procedure for limiting input traffic, which confirmed the effectiveness of using the proposed sharing architecture to improve the quality of transmission, especially in overload mode.

\section{Discussion}

The international standard "Media independent handover" [15] formalizes the VHO procedure. However, the implementation method remains open.

Using a decentralized last-mile access architecture using VHO by selecting the most appropriate of all possible networks using terminal device hardware and software has the following disadvantages: 
- Increasing the cost of terminal devices that must implement many protocols and contain special software that can form the criteria and selection procedures;

- Increasing complexity of terminal devices in the process of developing new and new technologies, standards and access procedures, the need to replace functional terminal devices with new ones due to the emergence of new technologies;

- Complex algorithms for interaction of network elements and, as a result, intensive exchange of service traffic using last-mile resources. For example, on LTE networks, service traffic exceeds the intensity of user traffic;

- Increase in energy consumption of terminal devices, which is unacceptable for devices with Autonomous power supply, such as sensory devices of the Internet of things.

A decentralized architecture based on the use of a complex terminal multifunctional device Software Defined Radio (SDR) has been studied in [13,14]. The task of the SDR was to collect data on the status of available access networks and their ability to provide the required quality of service in the last mile, and to select a network that is capable of providing high-speed data transfer modes.

VHO organization using SDR is only one of the solutions to the problem of choosing a network for access to global infocommunication resources. There is no alternative to this approach in special purpose networks that function in the interests of civil defense, state security, and law enforcement [16]. In these cases, the autonomy and efficiency of communication that terminal devices support is more important than their cost. At the same time, public access networks do not need complex and expensive terminals. Efficiency will only increase if terminal devices become cheaper and do not need to be replaced when new communication technologies become available. The centralized access architecture creates enlarged pools of network resources and improves multiplexing, multiple access, and switching technologies.

In contrast to those mentioned, this study sets out requirements for a centralized architecture for selecting a last-mile access network (see Figure 1). It involves adding a new aggregating device to the network elements of a heterogeneous access network, which is able to comprehensively analyze the state of all available access networks, terminal devices and existing requests for communication services. Access control turns into choosing the best network for each communication session at every moment in time at every point in space.

The $\mathrm{AD}$ is connected to all terminals and access networks and provides:

- Saving and analyzing data about available wireless access networks;

- Making decisions about choosing the best network according to the available criteria for all communication sessions;

- Dynamic reallocation of network resources between active terminals during their movement in space;

- Overload protection of individual networks,

- practical implementation of the ABC concept.

An aggregating device, every time when deciding on a suitable installed for connection of radio access, is based on existing consolidated data collected from all available access networks in a coverage area which falls terminal device and the type of requested service. In this case, the requirements for hardware and software components of terminals are less than in the decentralized approach. It is assumed that each terminal device supports a typical interface for interacting with the network aggregator. In turn, the $\mathrm{AD}$ on the terminal device side supports the standard interface, and on the base station side all the interfaces of available radio networks.

Advantages of the proposed centralized approach to selecting the last mile access radio network:

- Simple and inexpensive terminal devices-less stringent requirements for hardware and software resources of terminals than with a decentralized approach;

- The volume of service traffic is less than in the case of a decentralized approach;

- As terminal devices can be not only smartphones (people), but also smart things; 
- Corresponds to the national 5G launch programs, which involve: combining the resources of Telecom operators in the last mile, forming telecommunications ecosystems, and focusing on simple and inexpensive terminals [17];

- Meets the requirements for future infocommunications formulated in the Future Network concept of IEEE 3001 [18].

\section{Conclusions}

The proposed architecture of protocols for interaction between terminal devices and the base station and the strategy for selecting a network for access to global infocommunication resources meet the laws of development of telecommunication networks. The study confirmed the feasibility of creating centralized resources that provide access to various networks in the last mile.

The organizational and economic processes that can be observed today in the Russian mobile market suggest the possibility of creating a joint infrastructure owned by different Telecom operators to improve the efficiency of using the last mile resources and improve the quality of user service.

The proposed models can be applied to evaluate the characteristics of the quality of user service by networks of the 5 th generation.

Author Contributions: The research reported in this study was carried out by N.V., M.K. and A.S. Conceptualization, M.K. and N.V.; methodology, M.K.; software, N.V.; validation, N.V., M.K. and A.S.; formal analysis, N.V.; investigation, N.V.; resources, A.S.; data curation, A.S.; writing-original draft preparation, N.V.; writing-review and editing, M.K.; visualization, N.V.; supervision, M.K.; project administration, A.S. All authors have read and agreed to the published version of the manuscript.

Funding: This research received no external funding.

Conflicts of Interest: The authors declare no conflict of interest.

\section{References}

1. Verzun, N.; Kolbanev, M.; Vorobeva, D. Model of a Centralized Strategy for Selecting the Last Mile Access Network. In Proceedings of the 11th Majorov International Conference on Software Engineering and Computer Systems, Saint Petersburg, Russia, 12-13 December 2019.

2. Roadmap for the Development of "end-to-end" Digital Technology "Wireless Technology". 2019. Available online: https:/ / digital.gov.ru/uploaded/files/07102019vrar.pdf (accessed on 25 April 2020).

3. Gustafsson, E.; Jonsson, A. Always best connected. IEEE Wirel. Commun. 2003, 10, 49-55. [CrossRef]

4. Davalos, G.A.; Escobar, L.; Navarro, A. Vertical handoff algorithms-A new approach for performance evaluation. In Proceedings of the IEEE Globecom 2010 Workshop on Ubiquitous Computing and Networks, Miami, FL, USA, 6-10 December 2010; pp. 1724-1728.

5. Yan, X.; Sekercioglu, A.; Narayanan, S. Y. A survey of vertical handoff decision algorithms in Fourth Generation heterogeneous wireless networks. Comput. Netw. 2010, 11, 1848-1863. [CrossRef]

6. IEEE 802 LAN/MAN Standards Committee. Available online: http://www.ieee802.org/ (accessed on 25 April 2020).

7. Network 2030-A Blueprint of Technology, Applications and Market Drivers Towards the Year 2030 and Beyond; FG-NET-2030; Futurewei Technologies, Inc.: Santa Clara, CA, USA, 2019.

8. Verzun, N.A.; Kolbanev, M.O.; Omelyan. A.V. Controlled multiple access in wireless network of smart things.Omsk. Sci. Bull. 2016, 4, 147-151.

9. Bogatyrev, V.A.; Bogatyrev, S.V.; Bogatyrev, A.V. Model and Interaction Efficiency of Computer Nodes Based on Transfer Reservation at Multipath Routing. In Proceedings of the 2019 Wave Electronics and its Application in Information and Telecommunication Systems (WECONF), St. Petersburg, Russia, 3-7 June 2019.

10. Bogatyrev, A.V.; Bogatyrev, S.V.; Bogatyrev, V.A. Analysis of the timeliness of redundant service in the system of the parallel-series connection of nodes with unlimited queues. In Proceedings of the 2018 Wave Electronics and its Application in Information and Telecommunication Systems (WECONF), St. Petersburg, Russia, 26-30 November 2018. 
11. Astakhova, T.; Verzun, N.; Kolbanev, M.; Shamin, A. A model for estimating energy consumption seen when nodes of ubiquitous sensor networks communicate information to each other. In Proceedings of the 10th Majorov International Conference on Software Engineering and Computer Systems, Saint Petersburg, Russia, 12-13 December 2019.

12. Astakhova, T.N.; Verzun, N.A.; Kasatkin, V.V.; Kolbanev, M.O.; Shamin, A.A. Sensor network connectivity models. Informatsionno Upr. Sist. 2019, 5, 38-50. [CrossRef]

13. Grebeshkov, A.Y. Method of selecting a communication network to provide a service using a multifunctional subscriber device. Electrosvyaz 2011, 4, 53-55.

14. Grebeshkov, A.Y. Making a decision to provide a service with using a multifunctional SDR subscriber terminal in cognitive communication networks. In Proceedings of the VI Branchscientic and Technical Conference "Information society Technologies", Moscow, Russia, 3-4 March 2012; pp. 120-124.

15. IEEE Standard for Local and Metropolitan Area Networks-Part 21: Media Independent Handover. Available online: http:/ / www.ieee802.org/21 (accessed on 25 April 2020).

16. O svyazi [About the Connection]; Federal Law of Russia; Russian Government: Moscow, Russia, 2003.

17. 5G na Poroge. Available online: https:/ / www.rspectr.com/articles/521/5g-na-poroge (accessed on 25 April 2020).

18. Y 3001: Objectives and Design Goals. Available online: https://www.itu.int/itu-t/recommendations/rec. aspx?rec=11083 (accessed on 25 April 2020).

(C) 2020 by the authors. Licensee MDPI, Basel, Switzerland. This article is an open access article distributed under the terms and conditions of the Creative Commons Attribution (CC BY) license (http:// creativecommons.org/licenses/by/4.0/). 